De quelques enjeux et usages historiques du Français fondamental

\title{
Les applications du Français fondamental en contexte turc : aperçu historique (1966-1982)
}

Hanife Güven

\section{(2) OpenEdition}

\section{Journals}

Édition électronique

URL : https://journals.openedition.org/dhfles/1214

DOI : $10.4000 /$ dhfles. 1214

ISSN : 2221-4038

Éditeur

Société Internationale pour l'Histoire du Français Langue Étrangère ou Seconde

Édition imprimée

Date de publication : 1 janvier 2006

Pagination : 215-233

ISSN : 0992-7654

Référence électronique

Hanife Güven, « Les applications du Français fondamental en contexte turc : aperçu historique

(1966-1982) », Documents pour l'histoire du français langue étrangère ou seconde [En ligne], 36 | 2006,

mis en ligne le 06 septembre 2011, consulté le 27 mai 2021. URL : http://journals.openedition.org/ dhfles/1214; DOI : https://doi.org/10.4000/dhfles.1214

Ce document a été généré automatiquement le 27 mai 2021.

(c) SIHFLES 


\title{
Les applications du Français fondamental en contexte turc : aperçu historique (1966-1982)
}

\author{
Hanife Güven
}

\section{Introduction}

1 Le Français fondamental a été conçu comme un instrument qui donnerait aux apprenants étrangers une maîtrise minimale de la langue française en en assurant une diffusion rapide et solide. Il faisait état de l'intention de mettre davantage l'accent sur l'utilisation de la langue orale et des données probantes dégagées et fournissait un contenu lexical (selon les critères de fréquence et disponibilité), thématique (centres d'intérêt) et grammatical (assimilation des verbes irréguliers, maniement de l'interrogation avec " est-ce que » ou par l'intonation, inutilité relative de certains temps verbaux etc.).

2 L'histoire du Français fondamental en Turquie est directement liée aux actions entreprises par le Conseil de l'Europe ${ }^{1}$ en faveur des langues vivantes dans les pays membres. Commencée en 1966 pour l'anglais, le français et l'allemand, l'année précise où N. Chomsky (Chomsky $1978: 49-57)$ remettait en cause les bases linguistiques et psychologiques des méthodes audio-visuelles. L'échec relatif de ce renouvellement méthodologique est en partie lié aux théories sous-jacentes aux pratiques développées. En effet, quand la série de six ensembles audio-visuels traditionnels a été complétée en 1979, pour les six niveaux de l'enseignement secondaire, les méthodes communicatives avaient déjà commencé à révolutionner le domaine de la didactique des langues. Mais cela n'empêche pas de constater le succès indiscutable du projet.

Dans cette communication, il est utile de tracer d'abord le contexte historique en parcourant les étapes importantes, pour mieux comprendre le phénomène et le renouvellement qu'il a représenté dans le panorama scolaire et universitaire. Dans un deuxième temps, on verra rapidement les principes déclarés dans les préfaces de la 
série Je Parle français, l'unique méthode de français utilisée dans la Turquie de l'époque, créée à partir du Français fondamental et la formation des enseignants qui a accompagné cette entreprise. Nous voudrions finir notre propos avec quelques perspectives.

\section{Le contexte historique}

4 Pour réformer l'enseignement des trois langues vivantes dans l'enseignement secondaire général (l'anglais, le français, et l'allemand) le premier pas se fait avec la réunion des experts des Etats membres sur Les manuels de langues vivantes dans l'enseignement secondaire général à Ankara (6-16 septembre 1966). Ils adoptent une recommandation relative à la coopération internationale en vue de réviser les manuels utilisés, et surtout créer « un organisme chargé de promouvoir et de coordonner d'une part les travaux de recherche, de documentation et d'information et d'autre part, les activités de production de matériels d'enseignement ». Cette réunion fait suite à celle tenue à Ostie quelques mois avant (Avril 1966) sur Les examens terminaux de langues vivantes dans l'enseignement secondaire exposant en détail le contenu, et les niveaux stades initial - élémentaire -intermédiaire - avancé (EGT (66) Stage XXIV, 2). Juste avant la réunion d'Ankara, pour chacune des trois langues, les experts de deux pays (celui de la langue dont il s'agit et un pays où cette langue est enseignée dans de bonnes conditions) étudient les manuels scolaires en vigueur à l'époque. Pour les manuels de français les experts viennent de France et de Hollande, pour ceux d'anglais ce sont des experts du Royaume-Uni et de Norvège ; enfin les manuels d'allemand sont examinés par des experts de République Fédérale d'Allemagne et de Suède.

5 A l'époque, le français fait partie des trois langues vivantes étudiées dans l'enseignement secondaire. L'horaire hebdomadaire est de 3 heures dans le premier cycle. En revanche, dans le deuxième cycle, les périodes d'enseignement changent suivant les sections : alors qu'il est enseigné à raison de cinq heures hebdomadaires dans la section de littérature, dans les sections de sciences en première année à raison de cinq heures également, les deux années suivantes il n'y a que quatre cours hebdomadaires, chacun durant 40 à 45 minutes. Une seconde langue vivante à raison de deux heures par semaine est offerte aussi comme matière optionnelle. L'effectif des classes s'élève à 50 . L'élaboration des manuels est confiée au secteur privé mais c'est le Ministère de l'Education qui définit les critères et les adopte pour une durée de trois ans, renouvelable. Le contenu est strictement contrôlé par une équipe ad hoc du Ministère. Ils regroupent des textes fabriqués pour la première année du premier cycle de l'enseignement, mais au cours des années ils sont remplacés par des extraits littéraires édifiants. L'objectif prioritaire de l'apprentissage des langues est de fournir une ouverture littéraire et culturelle (DECS/EGT (70) 68).

6 Mais la prise de conscience de l'inefficacité de ces manuels dans un monde qui commence à changer semble avoir convaincu les décideurs politiques. La deuxième réunion tenue à Ankara en 1971 avec comme intitulé L'intégration des auxiliaires audiovisuels dans l'enseignement des langues vivantes en classe permet de faire un bilan des travaux en cours et d'en prévoir d'autres sur le plan gouvernemental, intergouvernemental et auprès des organisations non gouvernementales. A partir de cette année-là, le Ministère turc de l'Éducation Nationale lance une vaste campagne destinée à former les enseignants. On y reviendra plus tard. La création, l'année 
suivante, par décret ministériel de Yabanci Diller Ogretimini Gelistirme Merkezi, un Centre de Langues Vivantes, dépendant de la Direction de l'Education et de l'Enseignement (Talim ve Terbiye Kurulu) est un tournant décisif pour la modernisation de l'enseignement/apprentissage des langues étrangères. En effet, toutes ces préparations débouchent sur un nouveau programme qui sera publié dans le Bulletin officiel du Ministère de l'Education nationale (MEB Tebliğler Dergisi no : 1747 du 4 juin 1973) dans lequel on indique la mise en place de nouvelles méthodes pour subvenir aux besoins réels et la décision "d'appliquer graduellement le nouveau programme à partir de l'année scolaire 1973-1974 à commencer par la sixième année et de substituer toujours graduellement, à partir de l'année scolaire 1975-1976, les anciens manuels de langues vivantes écrits selon les programmes anciens. »

7 Les raisons de cette modification sont d'abord expliquées par rapport à la situation mondiale : « l'insatisfaction des résultats obtenus dans le monde entier par rapport au temps et à l'effort déployés pour enseigner les langues vivantes ». Les résultats médiocres étant fondamentalement dus à la méthodologie traditionnelle, il faudra proposer de nouvelles pistes avec de nouveaux objectifs :

Le sujet a été étudié par les spécialistes et on a développé de nouvelles méthodes et des instruments ayant pour but de développer la capacité de la compréhension orale et de parler à la place de l'ancien système classique d'enseignement des langues vivantes basé sur l'apprentissage par cœur des règles de grammaire et la traduction.

8 Après avoir posé ainsi le problème à l'échelle mondiale, voyons le projet mis en place en Turquie en collaboration avec le Conseil de l'Europe, en mettant en évidence la nouvelle politique éducative et linguistique qui constitue un choix entre tradition et modernité :

«Parallèlement à ces travaux internationaux, dans notre pays le sujet a été sérieusement traité lors de deux colloques organisés avec la collaboration du Conseil de l'Europe et, prenant en considération les sacrifices apportés dans cet objectif et l'attention toujours grandissante de notre peuple, il a été adopté un nouvel enseignement dont le résultat est bien plus fructueux. Pour cela, les programmes d'études des langues étrangères ont été révisés et, pour atteindre les objectifs indiqués dans les programmes, les commissions ad hoc créées par le Ministère de l'Education Nationale ont commencé la préparation du matériel nécessaire pour l'enseignement de l'allemand, du français et de l'anglais. »

On énumère cinq objectifs dont quatre sont les quatre compétences dans l'ordre connu : compréhension orale, compréhension écrite, expression orale, expression écrite. Le cinquième explicite l'objectif social très limité par ailleurs :

« Aider (les élèves) à se former (...) comme un acteur social en ce qui concerne les domaines du tourisme et des relations extérieures, en (leur) faisant acquérir la volonté et l'envie de développer et d'approfondir la langue apprise, après les études scolaires. »

Avec cette instruction, on officialisait le nouveau programme avec ses lignes directrices qui incluaient les objectifs, les auxiliaires à utiliser, la méthodologie et les niveaux. Le Projet Majeur Langues Vivantes du Conseil de la Coopération Culturelle du Conseil de l'Europe se concrétisera ainsi par la conception des méthodes An English Course for Turks pour l'anglais, Wir lernen Deutsch pour l'allemand et la méthode Je parle Français pour le français qui ont remplacé graduellement les anciens manuels. 


\section{La naissance de la méthode Je Parle français}

11 L'unique méthode pédagogique élaborée sur le terrain dans les années 70 en Turquie s'intitule Je Parle français. C'est une série de 6 ensembles pédagogiques, chacun correspondant aux six années/niveaux de l'enseignement secondaire public, destinée à des élèves de 12 à 17 ans. Chaque ensemble doit comprendre un livre du maître, un livre de l'élève, un cahier d'exercices et des tests. Mais étant donné la lenteur du rythme et l'ampleur de la tâche, et surtout la prise de conscience des changements théoriques, les matériels annexes et complémentaires n'ont pas pu être réalisés pour tous les niveaux. Le calendrier arrêté pour les différentes étapes de mise en œuvre est le suivant :

\begin{tabular}{|l|l|l|l|l|}
\hline Méthode/ année & Production & Expérimentation & Evaluation & Mise en place \\
\hline \hline JPF1 & $1970-1971$ & $1971-1972$ & $1972-1973$ & $1973-1974$ \\
\hline JPF 2 & $1971-1972$ & $1972-1973$ & $1973-1974$ & $1974-1975$ \\
\hline JPF3 & $1972-1973$ & $1973-1974$ & $1974-1975$ & $1975-1976$ \\
\hline JPF4 & $1973-1974$ & $1974-1975$ & $1975-1976$ & $1976-1977$ \\
\hline \hline JPF5 & $1974-1975$ & $1975-1976$ & $1977-1978$ & $1977-1978$ \\
\hline \hline JPF6 & $1975-1976$ & $1976-1977$ & $1978-1979$ & $1978-1979$ \\
\hline
\end{tabular}

Tableau 1. Calendrier arrêté pour l'élaboration des six ensembles

De même, le nombre de mots nouveaux à introduire a été déterminé pour chaque année et ensuite approuvé et intégré tel quel dans le Bulletin Officiel. Ahmet Erbaş qui assumait la fonction de responsable de la section de français auprès du Ministère de l'Education Nationale et qui avait participé à ce titre à toutes les étapes de la création de ces méthodes nous confiait récemment en ces termes le travail entrepris quant au lexique :

«Avant de procéder à la conception du matériel, la commission des manuels a établi des listes de vocabulaire et de structures grammaticales en s'aidant de la liste de fréquence par ordre décroissant du Français fondamental et cochait chaque mot utilisé, pour savoir par la suite combien de fois était utilisé chacun des mots prévus. Dans les quatre premières années (JPF1, 2, 3, 4), il a été rigoureusement tenu compte des connaissances passives que l'élève doit mettre en œuvre quand il les retrouvera dans des textes ou les entendra, et des connaissances actives qu'il est nécessaire de savoir réutiliser effectivement à l'oral ou à l'écrit dans des contextes nouveaux. Le contenu du Français fondamental 1 faisait partie des connaissances actives. Pour ce qui est du FF2 la commission a opéré des choix en tenant compte des consignes qui l'accompagnent » (Entretien réalisé en novembre 2005).

\begin{tabular}{|l|l|l|l|}
\hline Stade & Classe & \multicolumn{2}{|l|}{$\begin{array}{l}\text { Nombre de mots prévus } \\
\text { Au moins Au plus }\end{array}$} \\
\hline Stade initial et stade élémentaire 1 & Moyenne 1 & 200 & 200 \\
\hline
\end{tabular}




\begin{tabular}{|l|l|l|l|}
\hline Stade élémentaire 2 & Moyenne 2 & 250 & 300 \\
\hline Stade intermédiaire 1 & Moyenne 3 & 250 & 300 \\
\hline Stade intermédaire 2 & Lycée 1 & 300 & 350 \\
\hline Stade avancé 1 & Lycée & 300 & 350 \\
\hline Stade avancé2 & Lycée & 300 & 350 \\
\hline Total & & 1550 & 1800 \\
\hline
\end{tabular}

Tableau 2. Nombre de mots à introduire pour chaque niveau d'enseignement Source : Bulletin Officiel $N^{\circ} 1747$ du 4 juin 1973

\section{Les fondements de la méthode}

Dès le début de 1968 le comité constitué avait fixé les fondements sur lesquels se baserait la nouvelle méthode de français en ces termes :

«Le comité envisage de commencer par la méthode audiovisuelle, à l'aide d'enregistrements sur magnétophone ainsi que de tableaux de feutre et de figurines comme principal moyen de présentation visuelle. Les trois premiers mois seront entièrement consacrés à l'enseignement oral avec un vocabulaire limité. Pour le choix des points lexicologiques, l'ouvrage Le Français fondamental servira de guide et la sélection des structures tiendra compte de L'Inventaire syntagmatique du Français fondamental, publié par le BELC. Un guide du professeur sera utilisé ainsi que des manuels des élèves pour la période suivant les trois premiers mois. En outre, une série de tests seront mis au point pour la première année. Il est prévu de faire essayer le matériel dans certaines écoles avant de lui donner sa forme définitive " (CCC/EGT (70) 10)

14 En effet on est frappé de constater la similitude des principes déclarés et des consignes utilisées dans JPF $1^{2}$ avec ceux des méthodes SGAV élaborées en France, notamment Voix et images de France, à commencer par l'avant-propos jusqu'aux termes utilisés. Les principes fondamentaux sous-jacents à la méthode se retrouvent dans la première partie de l'avant-propos où sont exposés les fondements de la méthode dans lesquels on reconnaît les préoccupations essentielles :

«- de toute évidence, toutes les langues se parlent avant de s'écrire.»

«-le langage ne se développe d'autre part, naturellement, qu'en situation.»

Dans cette perspective privilégiée, les auteurs affirment qu'« une priorité absolue sera tout d'abord accordée au français " oral " ", le mot oral étant mis entre guillemets. On trouve deux autres mots entre guillemets dans le paragraphe qui suit : « situation » et « dialogue » :

«Le caractère naturel de cette langue parlée sera obtenu par la recherche des réparties effectivement utilisées dans la vie de tous les jours (sic). Cette constatation conduit à choisir les "situations " les plus courantes, c'est-à-dire, celles qui sont susceptibles de faire jaillir " le dialogue " le plus vraisemblable.

Afin de faire entrer la vie dans la classe, il conviendra alors d'illustrer ces " situations » et de faire parler l'image grâce à l'enchaînement du geste et de la parole.» 
Dans une culture encore fortement marquée par l'hégémonie des textes modèles édificateurs, du poids de l'écrit, des modes d'évaluation classique, il est facile de constater le souci des auteurs pour introduire des concepts aussi nouveaux. En plus des mots entre guillemets, on trouve plus loin cinq mots en caractères gras :

" (la méthode) fait en effet appel à l'audition d'un dialogue.[...] Elle est visuelle dans la mesure où l'image (figurines de carton floqué sur feutrine ${ }^{3}$ ) demeure support et générateur de dialogues (et non de descriptions). Elle est structurale parce que l'image invite automatiquement et spontanément l'élève à s'exprimer dans une langue étrangère, soit à utiliser les véritables structures du français sans passer par la réflexion ou l'exercice de traduction [...].»

Les deux derniers paragraphes font référence à la « progression » et au " contrôle » en ce sens que la progression strictement liée aux indications données au début de chaque dossier assurera aussi un contrôle plus efficace. Le socle du renouveau, le Français fondamental, semble quasiment passé sous silence en ce sens qu'il n'est ni souligné, ni mis entre guillemets :

Il convient de mentionner qu'il a été tenu compte de l'âge des élèves dans le choix des centres d'intérêt (contenu thématique des dialogues) et du Français fondamental ${ }^{4}$, inventaire des termes les plus fréquemment utilisés dans la vie courante.

L'avant-propos de JPF2 est consacré au troisième moment de la classe audio-visuelle qui est l'expression libre, avec beaucoup d'explications et surtout beaucoup d'exemples sur des exercices de réemploi et d'expression spontanée. L'utilisateur est d'emblée invité à relire l'avant propos de JPF1 « les principes et la méthode qui ont présidé à la rédaction du manuel II étant les mêmes que ceux appliqués dans le manuel I ». Plus loin, on est frappé de la focalisation sur l'aspect statistique de l'entreprise qui devient presqu'une obsession :

«On trouvera à la fin du livre II du maître et de l'élève une liste dans l'ordre alphabétique du lexique utilisé dans les manuels I et II (289 pour le I et 301 pour le II). Il faut savoir que le Français fondamental comprend 1500 mots dont seuls environ 1000 sont employés fréquemment par les Français eux-mêmes. »

En effet, à la fin du livre on trouve une liste de mots avec l'indication de la leçon qui les introduit. De même dans JPF 3, on porte toujours une attention au nombre de mots introduits qui est prévu à 350, mais on attire l'attention sur le fait que, si on compte les mots composés à partir de deux éléments connus (mal + heureux), les mots " phonétiquement identiques aux mots turcs ", les adjectifs dont le radical est déjà connu, le nombre de mots de vocabulaire peut être estimé à environ 300. Il est précisé que « la langue orale est toujours prépondérante néanmoins l'écrit y tient une place de plus en plus importante ».

22 JPF4 est prévu pour la première année du lycée qui accueille des élèves provenant de divers établissements ou régions dans lesquels la qualité de l'enseignement n'est pas toujours égale. L'objectif primordial est alors de niveler les inégalités et de permettre aux élèves de se trouver à chances égales. Mais on est en 1976, et, dans le monde de la didactique, on constate l'émergence des documents authentiques, des unités capitalisables du Conseil de l'Europe, des actes de parole. La publication de C'est le Printemps change les thèmes introduits en en proposant de plus problématiques. Avec De vive voix, la méthodologie avait déjà commencé à devenir plus intégrée. Ce travail de longue haleine ne pouvait pas se tenir à l'abri des nouvelles orientations 
méthodologiques. Il est intéressant de constater l'importance accordée à l'authenticité et la véracité des dialogues et des textes :

«Les leçons sont présentées sous une forme entièrement nouvelle. Nous avons délibérément écarté un français stérilisé, désuet, souvent réservé aux étrangers en nous demandant chaque fois, si des élèves français ne souriraient pas à la lecture de nos dialogues et de nos textes.

L'élève de quatrième année sera donc confronté à la langue française d'aujourd'hui dans un cadre contemporain. »

Avec ce 4e niveau, on épuise le Français fondamental 1er degré, mais le FF2 est systématiquement repris ; avec JPF5 et JPF6 on continue à utiliser le lexique mais on trouve beaucoup plus de documents authentiques dans les manuels et le poids de l'écrit augmente.

Dans le but de donner un élan supplémentaire à la campagne lancée, on élabore cinquante-deux émissions de radio de 15 minutes chacune, et vint-sept émissions de télévision en mettant en place des canaux de diffusion qui dépassent les cercles de classe et qui touchent ainsi un public plus large.

\section{La formation initiale et continue des enseignants}

Les effets internes d'une entreprise aussi importante concernaient une série de réformes interdépendantes, dont la plus cruciale était la formation, notamment des maîtres formés avec les méthodologies traditionnelles. En Turquie, la formation des maitres de langues étaient assurée, jusqu'à l'année scolaire 1960-1961, dans les Instituts Pédagogiques où la durée des études était de deux ans; passée à trois ans à partir de cette date, il faudra attendre l'année scolaire 1978-79 pour que la formation initiale passe à quatre ans et que les institutions se substituent désormais aux Écoles Normales Supérieures. Mais entre les années 1975-1980, le pays a vécu une déstabilisation économico-politico-sociale qui a influencé aussi la formation des enseignants. Les jeunes se voyaient offrir des formations intitulées " cours du soir ", " cours par correspondance " et " cours accélérés " et recevaient les diplômes de futurs enseignants sans trop se familiariser avec les divers aspects du métier. Non seulement les maitres formés avec les méthodes traditionnelles, mais aussi et surtout ces jeunes diplômés avaient besoin d'être préparés à affronter les nouveaux enjeux. D'où une vogue d'intérêt inattendue pour la formation continue, comme cela apparaît dans les avant-propos des manuels, les séminaires nationaux et régionaux, et la revue de liaison de maîtres, mais aussi dans des séminaires pour les formateurs des formateurs et dans l'attribution de bourses.

\subsection{Séminaires nationaux et régionaux pour les enseignants}

Une vaste campagne a été lancée par le Ministère pour « former tous les professeurs de français aux méthodes modernes en suivant de près un calendrier de formation et en commençant par les professeurs enseignant dans le premier cycle de l'enseignement secondaire " (Erbas 1981: 29). Le succès de la campagne entreprise pour initier les enseignants à cette nouvelle conception de l'enseignement est tel que cinquante et un stages d'été seront organisés à partir de l'année 1970, à commencer par ceux qui sont destinés à former les expérimentateurs des vingt-sept établissements pilotes. Deux 
mille quatre cents quarante cinq professeurs de français sont formés entre 1970-1983 (DECS /EGT (83) 41). référence a été créée dans le domaine. La vie de Rencontre n'a pas été longue. Elle a été publiée sous forme de sept numéros entre 1975-1984 dont le dernier s'intitule Rencontres, désormais avec un « $\mathrm{s}$ ». Ce passage d'un intitulé singulier à une notion plurielle marque une approche d'ordre sociologique qui annonce, selon nous, la diversité et le dialogue au sens d'aujourd'hui. Destinée aux professeurs de français langue étrangère et distribuée gratuitement dans les écoles, elle constituait un bulletin de liaison et plaidait surtout la cause de l'oralité, en présentant les nouvelles méthodes, les divers moments d'une leçon audio-visuelle, l'évaluation, l'utilisation des documents authentiques. Si on pense aux difficultés économiques des enseignants à se procurer le Français dans le Monde elle comblait à sa façon, une lacune importante dans un environnement lointain et permettait au professeur de français de trouver une référence et parfois une réponse à ses interrogations. Sa contribution à mettre en route une nouvelle conception de l'enseignement est évidente même si ses effets sont limités.

\subsection{Séminaires pour les formateurs des formateurs}

Après les tout premiers séminaires qui ont lieu début 70, à la fin de la décennie on en envisage trois relatifs à la formation des formateurs suivant les directives du Conseil de l'Europe qui recommande au comité des Ministres :

«d'inviter les gouvernements des États membres à intensifier leur coopération bilatérale avec la Turquie dans le domaine de l'enseignement des langues vivantes, notamment par l'envoi d'experts et l'octroi de bourses à des professeurs, ainsi qu'à des administrateurs chargés de la mise en œuvre du Plan, susceptibles à leur retour de contribuer par un effet multiplicateur, à la formation d'enseignants. »

Ils sont organisés respectivement en 1980 à Izmir, en 1981 à Bursa et en 1982 à Ankara afin de familiariser les acteurs principaux de la formation avec les notions de base qu'ils doivent connaître et transmettre ensuite aux futurs enseignants. Tous les formateurs inscrits sont tenus d'y assister. Il suffit de citer les noms de G. Khan et $\mathrm{H}$. Holec qui ont participé au stage d'Izmir, de M. J. Gremmo et F. Carton qui ont animé celui d'Ankara, pour illustrer la préoccupation à offrir un contenu pluridisciplinaire, une amélioration des curricula, et surtout à faire évoluer les mentalités en élargissant les champs de références éducatives. Les cours théoriques et pratiques présentés sous forme d'ateliers portent sur les sujets suivants : cours de langue, Français fondamental, notion de structure, passage à l'écrit, langue orale, lange écrite, les divers moments d'une classe de langue, présentation des manuels Je Parle français, évaluation et 
autoévaluation, nouvelles tendances dans l'enseignement /apprentissage des langues etc.

\section{Les limites}

On a souligné plus haut l'enthousiasme des professeurs pour la nouvelle méthode considérée par eux comme synonyme d'une modernité. Les difficultés rencontrées proviennent en partie de la conjoncture politique et économique morose. Le diagnostic émanant du projet de recommandation dit à lui seul l'ampleur mais aussi la nécessité de l'entreprise à affronter :

«De tous les États membres du Conseil de l'Europe, c'est certainement la Turquie qui doit faire face au plus grand nombre de difficultés si elle veut que ses citoyens apprennent au moins une langue européenne de grande diffusion; d'une part la langue turque, d'origine ouralo-altaïque, a très peu de choses en commun avec les autres langues parlées dans les Etats membres du Conseil de l'Europe, ce qui implique un effort plus grand de la part des citoyens turcs désireux d'apprendre des langues telles que l'anglais, le français et l'allemand; d'autre part son éloignement géographique, son industrie et son commerce relativement peu développés, ses problèmes d'alphabétisation et de scolarisation constituent des obstacles considérables. (Projet de recommandation, 594 (1970) Assemblée consultative). »

A cet état des lieux s'ajoutent autant de contraintes spécifiques qui appesantissent le climat local : l'image du français langue difficile, effectif très élevé des classes, en moyenne 50 élèves, horaire hebdomadaire limité, manque de matériel périphérique, centralisation extrême du système éducatif, poids limité de l'écrit qui constituait un manque de sérieux dans une société imprégnée de la conception « verba volant.... » et très liée aux pratiques anciennes et à l'aspect culturel transmis à travers les manuels. En 1982, les Ecoles Normales Supérieures sont rattachées au Conseil de l'Enseignement Supérieur et sont désormais "Facultés de Pédagogie". Cette mise en oeuvre de nouvelles politiques éducatives a des avantages considérables en ce sens que, d'une part la formation des maitres est désormais assurée au sein des universités et non plus gérée par le Ministère qui est parfois très politisé ; d'autre part les formateurs deviennent enseignants-chercheurs. Mais la rupture créée entre les universités et le Ministère, dans un premier temps, a constitué un obstacle pour le renforcement du suivi du projet.

\section{Perspectives}

A la lumière de ces observations, on pourrait affirmer que, étant donné le contexte historico-social, cette nouvelle méthode n'a pas apporté la relance espérée de l'enseignement $d u$ français dans les institutions scolaires publiques, mais le rôle clef que le Français fondamental accordait à la langue orale a facilité l'introduction, plus tard, d'une nouvelle phase plus efficace dans l'enseignement du français.

L'enthousiasme créé durant une quinzaine d'année en Turquie dans un contexte où l'on déplore toujours le " manque d'expression orale » (Erbas $1981: 30)$ des enseignants, cette nouvelle conception de l'enseignement/apprentissage avaient changé le paysage et remis en question non seulement les principes qui sous-tendaient les anciennes méthodes mais aussi les objectifs de l'apprentissage/enseignement des langues étrangères. 

progressive de départements spécialisés de français dans les universités parallèlement à des écoles ou départements bilingues qui dispensent un enseignement en français. La création du nouvel intitulé de la direction qui devient en 1988 YADEM (Centre pour le développement de la didactique des langues étrangères) est la preuve du rôle-clef accordé à la recherche pédagogique dans le domaine de la didactique des langues, dont la mission fondamentale sera l'incitation permanente à un certain dynamisme et l'amélioration des efforts engagés depuis 20 ans. En voici à titre indicatif, quelques exemples : le renouvellement permanent des manuels, la formation initiale et continue des enseignants, la prolongation de l'éducation de base qui durera huit ans, le commencement de l'enseignement des langues dès la quatrième année de l'enseignement primaire, l'instauration d'une seconde langue obligatoire. Mais il n'y a pas lieu d'être pessimiste si on prend en compte que la régression dans les établissements publics n'empêche pas la création de lycées bilingues privés ou publics concernant aussi bien l'enseignement général que professionnel, et qui garantissent un enseignement linguistique plus efficace. Ils sont au nombre de dix-neuf, à raison de neuf à Istanbul, six à Izmir et trois à Ankara. ${ }^{5}$ La caractéristique de certains de ces lycées est de former les élites du pays parfaitement francophones et ils ont une réputation de qualité au niveau national. Lors des cérémonies pour fêter le 150e anniversaire de sa fondation, le lycée Notre Dame de Sion fondé en 1850, se vante d'avoir formé les trois filles adoptives de Mustafa Kemal Atatürk, le fondateur de la République laïque.

progrès significatifs ont été accomplis aussi dans l'enseignement supérieur quant à la fonctionnalité de la langue enseignée. Parmi les établissements les plus fonctionnels, on peut citer avant tout l'Université de Galatasaray (1992) (avec le français comme langue d'enseignement) qui a toujours occupé une place importante dans la formation des élites (avec ses facultés de droit, de sciences administratives et économiques, de communication, des lettres et sciences et d'ingénierie un département des sciences politiques et relations internationales (Istanbul Yeditepe), un département d'administration publique (Istanbul Marmara), un département de langues avec l'allemand et l'espagnol (Istanbul Bosphore), cinq départements de traduction et interprétariat (Istanbul Yildiz, Ankara Hacettepe, Bilkent, Kirikkale, Mersin), et des centres d'éducation permanente qui réorientent le rôle culturel de la langue vers le commerce, le tourisme etc. en fonction de besoins réels mais aussi au service des autres disciplines.

A part cela, il existe onze départements de didactique de langue française qui forment les futurs professeurs de français au sein des facultés de pédagogie (Anadolu, Atatürk, Çukurova, Dicle, Dokuz Eylül, Gazi, Istanbul, Marmara, Konya, Samsun, Uludağ) et six départements de langue et littérature françaises au sein des facultés des sciences et lettres (Istanbul, Hacettepe, Ankara, Konya, Sivas, Erzurum). A part ces départements proprement dits, on trouve le français comme langue étrangère dans les universités Gazi (Ankara), Dokuz Eylul (Izmir), Süleyman Demirel (Isparta), Malatya, Kirikkale, Gazi Osman Paşa (Tokat), On sekiz Mart (Çanakkale) assuré par des centres ou écoles supérieures ou départements de langues étrangères (Antalya, Aydin) qui assurent la classe préparatoire, ou les cours optionnels ou obligatoires. Faute de public francophone à l'entrée en université, pour résister à la concurrence de l'anglais, ces 
institutions acceptent de recruter, depuis cinq ans, des étudiants qui ne connaissent pas le français.

Cependant on peut critiquer cette pratique de recrutement, car elle a des répercussions alarmantes pour les départements de didactique, qui ainsi perdent toute leur crédibilité en tant qu'institution de formation des maitres, elle semble ne contribuer qu'à une baisse de niveau. Dans l'état actuel, le public qui est extrêmement hétérogène semble seulement viser le diplôme. La maîtrise du français va du faux débutant au vrai bilingue, ce dernier étant beaucoup plus rare. La plus grande partie des futurs enseignants sont originaires des écoles bilingues anglophones et après une année préparatoire ils commencent à s'initier au métier. Les enseignants de formation littéraire restent très dominants. Et le bon sens y voit une forte incohérence. C'est aux responsables de ces départements à revoir leurs offres et à développer une stratégie de qualité pour ne pas continuer à être les départements "d'un autre siècle " comme le soulignait P. Dumont (Dumont 1999 : 48) avant déjà le changement du système de recrutement. Les cinq années d'expériences peuvent contribuer à ouvrir une nouvelle perspective en déterminant une nouvelle politique linguistique plus efficace. Il reste vrai cependant que l'évolution future dépendra en grande partie de la clairvoyance respective des décideurs et des formateurs de formateurs et de l'attrait que les institutions auront auprès du public.

Depuis l'année 1966, les politiques éducatives nationales turques ont porté une attention particulière au renouvellement des méthodes d'enseignement de langues et, en parallèle, à la formation des enseignants. Le rôle significatif que le Français fondamental y a joué, est celui d'avoir permis de dépasser des pratiques traditionnelles essentiellement fondées sur l'écrit. Il sert toujours de base aux nouvelles méthodes créées en Turquie, et reste un lieu de mémoire pour l'enseignement du français dans l'abandon des anciennes structures et la détermination d'une nouvelle politique éducative qui visait à aller de pair avec les pays d'Occident, l'Occident vers lequel est toujours tourné le visage de la Turquie moderne.

\section{BIBLIOGRAPHIE}

CHOMSKY, N. (1978), «Théorie Linguistique», La Pédagogie du Français langue étrangère, Ali Bouacha A. (éd), Paris : Hachette, pp. 49-57.

DUMONT, P. (1999), La politique linguistique et culturelle de la France en Turquie, Paris : L'Harmattan.

ERBAş, A. (1981), « Formation des professeurs de français en Turquie », In-service Training for the teachers of foreign languages in Turkey, Ankara : Ministry of National Education, Board of Education, pp. 27-39.

ERBAŞ, A. (1983), Rapport pour le nouveau Programme d'Assistance Multilatérale pour le développement des langues vivantes en Turquie, Strasbourg: Conseil de l'Europe DECS/EGT (83) 41.

Conseil Supérieur de l'Éducation Nationale, Centre de langues vivantes, Ankara. 
Je Parle français 1 Livre du maître Stade Initial- Stade Elémentaire 1(1977), Istanbul : MEB.

Je Parle français 2 Livre du maître Stade Elémentaire 2 (1975), Istanbul : MEB.

Je Parle français 3 Livre du maître Stade Intermédiaire 1 (1976), Istanbul : MEB.

Je Parle français 4 Livre du maître Stade Intermédiaire 2 (1978), Istanbul : MEB.

Je Parle français 5 Livre du maître Stade Avancé 1 (1979), Istanbul : MEB.

Je Parle français 6 Livre du maître Stade Avancé 2 (1979), Istanbul : MEB.

NEUMEISTER, H. (1970), Etude comparative sur l'enseignement des langues vivantes en Europe. Monographie sur la Turquie. DECS/ EGT (70) 68, Strasbourg : Conseil de l'Europe.

Rapport sur les manuels de langues vivantes dans l'enseignement secondaire général EGT (66) Stage XXIV, 2, Strasbourg : Conseil de l'Europe.

RlDDY, D.C., L'enseignement des langues vivantes en Turquie, CCC/EGT (70) 10 Révisé, Strasbourg : Conseil de l'Europe.

Symposium sur L'intégration des auxiliaires audio-visuels dans l'enseignement des langues vivantes en classe, CCC/EGT (72) 11, Strasbourg: Conseil de l'Europe.

\section{NOTES}

1. En effet, la Turquie a adhéré au Conseil dès l'année de sa fondation (le 5 mai 1949), en devenant le 13e État membre le 9 août 1949 et a signé en décembre 1954 la Convention culturelle européenne, cadre de la coopération du Conseil de l'Europe dans les domaines de l'éducation, de la culture, de la jeunesse et du sport, qui est entrée en vigueur en Turquie en 1957.

2. Les citations sont faites d'après l'édition de 1977 du livre du maitre

3. Les premiers temps, on a utilisé une feutrine et des figurines de carton floqué à la place du film fixe.

4. Souligné par nous

5. A Ankara, lycée privé Tevfik Fikret, lycée Anatolien d'Ankara, lycée Charles de Gaulle, à Istanbul, les lycées Galatasaray, lycée français de Notre Dame de Sion, lycée français privé Saint Joseph, lycée Pierre Loti, lycée français privé Saint Michel, lycée français Saint Benoit, lycée professionnel Anatolien de Kagithane Profilo, lycée Anatolien Burak Bora, lycée français Saint Pulchérie, à Izmir, lycée Ataturk, lycée Anatolien de Buca, lycée Anatolien de Bornova, lycée privé Tevfik Fikret, lycée privé Français Saint Joseph, lycée privé scientifique Tefvik Fikret.

\section{INDEX}

Mots-clés : français fondamental, Turquie, méthode pédagogique, formation des enseignants, politique éducative

Keywords : basic French, Turkey, educational methods, teacher training, educational policy 
AUTEUR

HANIFE GÜVEN

Université de Dokuz Eylül 